\title{
Research as Development
}





\title{
Research as Development
}

\author{
Biomedical Research, Ethics, and \\ Collaboration in Sri Lanka
}

Salla Sariola and Bob Simpson 


\section{Copyright () 2019 by Cornell University}

All rights reserved. Except for brief quotations in a review, this book, or parts thereof, must not be reproduced in any form without permission in writing from the publisher. For information, address Cornell University Press, Sage House, 512 East State Street, Ithaca, New York 14850. Visit our website at cornellpress.cornell.edu.

First published 2019 by Cornell University Press

Printed in the United States of America

Library of Congress Cataloging-in-Publication Data

Names: Sariola, Salla, author. I Simpson, Bob, 1956- author.

Title: Research as development : biomedical research, ethics, and collaboration in Sri Lanka / by Salla Sariola and Bob Simpson.

Description: Ithaca : Cornell University Press, 2019. I Includes bibliographical references and index.

Identifiers: LCCN 2018042027 (print) | LCCN 2018043945 (ebook) | ISBN 9781501733611 (e-book pdf) I ISBN 9781501733628 (e-book epub/mobi) I ISBN 9781501733604 | ISBN 9781501733604 (cloth; qalk. paper)

Subjects: LCSH: Medicine-Research—Sri Lanka. I MedicineResearch-International cooperation. I Clinical trials-Moral and ethical aspects-Sri Lanka. I Medical ethics-Sri Lanka. I BioethicsSri Lanka. I Medical economics-Sri Lanka.

Classification: LCC R854.S72 (ebook) | LCC R854.S72 S37 2019 (print) | DDC 610.7205493 — dc23

LC record available at https://lccn.loc.gov/2018042027 